\title{
Masked prime stimuli can bias "free" choices between response alternatives
}

\author{
FRIEDERIKE SCHLAGHECKEN \\ University of Warwick, Coventry, England \\ and \\ MARTIN EIMER \\ Birkbeck College, University of London, London, England
}

\begin{abstract}
Stimuli presented below the threshold of awareness can systematically influence choice responses determined by the instructed stimulus-response (S-R) mapping (task set). In this study, we investigated whether such stimuli will also bias a free choice between two response alternatives under conditions in which this choice subjectively appears to be internally generated and free. Participants had to respond to targets preceded by masked arrow primes. Left-pointing and right-pointing arrow targets required left or right responses, whereas randomly interspersed "free-choice" targets indicated that the participants were free to choose either response. Although masked primes could not be consciously discriminated, they systematically affected not only performance to arrow targets, but also the free choice between response alternatives. This demonstrates that apparently "free" choices are not immune to nonconsciously triggered biases. However, in blocks in which no specific S-R mapping was imposed, masked primes did not affect free-choice performance, indicating that these effects are not automatic but are determined by currently active task sets.
\end{abstract}

The possibility that stimuli that are not consciously perceived can affect a person's behavior is often regarded as a cause for concern. Could undetectable - and hence, uncontrollable - external forces bias what people like to regard as voluntary and free decisions? Could subliminal stimuli ${ }^{1}$ control actions that are independent of, or perhaps even contrary to, current intentions? Increasing evidence suggests that such nonconscious influences exist, with subliminal stimuli biasing subjectively "free" semantic and affective processing (see, e.g., Kihlstrom, 1999). In the present study, we investigated whether similar influences can be observed in the motor domainthat is, whether subjectively free motor responses are biased by stimuli presented below the threshold of awareness.

A masked prime paradigm was employed, in which participants had to execute motor responses to visual stimuli preceded by backward-masked visual primes. Recent studies have demonstrated that successfully masked primes can affect the motor system (e.g., Dehaene et al., 1998; Leuthold \& Kopp, 1998; Neumann \& Klotz, 1994). Although primes were presented below identification threshold, reaction times (RTs) and error rates differed depending on whether the prime-target relationship was

The present research was partly supported by a grant to M.E. from the Biotechnology and Biological Sciences Research Council. The experiment was conducted at the Institute of Applied Cognitive Science at the University of Warwick. Correspondence concerning this article should be addressed to F. Schlaghecken, Department of Psychology, University of Warwick, Coventry CV4 6AL, England (e-mail: f.schlaghecken@ warwick.ac.uk). compatible (both stimuli mapped to the same response), incompatible (each mapped to a different response), or neutral (prime not mapped to any response). Performance was facilitated on compatible trials and impaired on incompatible trials, relative to neutral trials (positive compatibility effect, $P C E$ ). Other experiments (Eimer, 1999; Schlaghecken \& Eimer, 1997, 2000) have demonstrated that such priming effects show a specific relationship to the interstimulus interval (ISI) separating masked primes and targets. PCEs were observed when targets followed the primes immediately but turned into negative compatibility effects ( $N C E \mathrm{~s})$, with performance benefits on incompatible trials and costs on compatible trials, when ISI was increased.

Two features of these subliminal priming effects are of special interest in the present context. First, NCEs are elicited only when primes are presented near or below the level of conscious awareness. There is a close correlation between priming effects and prime visibility, with NCEs observed for primes that could not be identified turning into PCEs when the primes become consciously available (Eimer \& Schlaghecken, 2002; Klapp \& Hinkley, 2002). Second, NCEs are not generated at perceptual or at central-semantic levels. They have been obtained with different types of primes, masks, and targets (Eimer, 1999; Eimer \& Schlaghecken, 1998) and even when primes and targets are physically dissimilar and appear at different locations (e.g., Schlaghecken \& Eimer, 2000), ruling out a perceptual explanation of NCEs. Moreover, NCEs do not exhibit any transfer of effects across response modalities: When, for example, primes specify 
responses with the left or right hand, only hand responses, but not foot responses, are affected by prime-target compatibility (Eimer, Schubö, \& Schlaghecken, 2002). This indicates that NCEs do not arise at the level of effectorunspecific semantic left or right codes.

This leaves the motor system as the most likely source of NCEs. Direct measures of motor cortex activity indicate that masked primes rapidly activate their corresponding motor response (Dehaene et al., 1998; Eimer, 1999; Eimer \& Schlaghecken, 1998; Leuthold \& Kopp, 1998). Target-related response preparation during this initial phase results in PCEs, since responses on compatible trials are already primed, whereas responses on incompatible trials conflict with the primed response tendency. When no target response is prepared during this phase, the initial activation not only decays, but becomes actively inhibited. Converging behavioral (e.g., Schlaghecken \& Eimer, 1997), electrophysiological (Eimer, 1999; Eimer \& Schlaghecken, 1998), and hemodynamic (Aron et al., 2003) evidence suggests that automatic self-inhibition mechanisms quickly suppress activity in the primed response channel below baseline levels. Response preparation during this inhibitory phase results in NCEs, since the response required on compatible trials is inhibited, whereas the alternative (i.e., the incompatible) response is disinhibited from its competitor (Schlaghecken \& Eimer, 2002).

Although these facilitatory and inhibitory components of subliminal priming apparently reflect low-level, automatic motor control processes, they are not independent from current intentions. Subliminal priming effects are observed only when primes match the currently active task set (the knowledge about target identity and $\mathrm{S}-\mathrm{R}$ mappings). For example, when primes are left- or right-pointing arrows, NCEs are observed when at least some of the targets are left- or right-pointing arrows as well. In contrast, when primes do not match the active task set (e.g., when arrows are used as primes but responses are assigned to letter targets), no priming is observed (Eimer \& Schlaghecken, 1998, Experiment 1B). It therefore seems unlikely that subliminal stimuli could affect overt behavior independently of or even contrary to current intentions.

However, initial evidence suggests that given an appropriate task set, masked primes may bias subjectively free response decisions (Klapp \& Hinkley, 2002, Experiment 5). Trials in which the response was determined by an arrow target (instructed trials) were mixed with trials in which response choice to a nonarrow target was free (free trials). All the trials employed masked arrow primes. Not only were priming effects on RTs similar for instructed and free trials, but primes also biased response choice on free trials, with noncongruent responses (responses executed with the hand not indicated by the prime) being chosen more frequently than congruent responses. However, primes were perhaps not strictly subliminal, since prime identification performance (tested in a different experiment under identical presentation conditions) was above chance.
The aim of the present experiment was to provide more substantive evidence for the hypothesis that subliminal primes can bias subjectively free choices between response alternatives. Its goal was to demonstrate that such biases (1) occur even when primes are clearly below identification threshold, (2) exhibit the same facilitationfollowed-by-inhibition pattern observed previously with PCEs at short prime-target ISIs and NCEs at longer ISIs, and (3) are determined by currently active task sets.

To investigate these issues, the participants had to perform three tasks. A forced-choice prime identification task was employed to demonstrate that identification performance for masked primes was at chance level. This task was administered before and after the main experiment, to determine whether prime identification improves with experience. A mixed RT task with randomly intermixed instructed trials and free trials was used to investigate whether subjectively free response decisions are biased by subliminal primes in the presence of a specific task set. Prime-target ISI in this task was blocked and was either 0 or $150 \mathrm{msec}$, expected to result in PCEs and NCEs, respectively, for instructed trials. If freechoice responses are susceptible to the same facilitationfollowed-by-inhibition processes, a similar pattern should be obtained: Congruent responses (corresponding to the primes' direction) should be faster and more frequent than noncongruent responses with a 0 -msec ISI, whereas the reverse should be true with a 150 -msec ISI.

Finally, a "pure" free-choice RT task (without instructed trials) was included to study whether priming effects on free response decisions can also be observed when no specific task set is imposed. Free-choice blocks were presented before and immediately after the mixed RT task, to test whether the existence of a recently active task set might change the primes' influence on free response choice. Prime-target ISI was again $0 \mathrm{msec}$ in half of the blocks and $150 \mathrm{msec}$ in the other half.

\section{Method}

Participants. Thirteen women and 11 men, 18-31 years of age (mean age, 23.1 years), participated. According to self-report, all but 1 were right-handed, and all had normal or corrected-to-normal vision.

Stimuli and Apparatus. The primes were left- and right-pointing double arrows $(<<$ and $>>)$. The targets were either left- and right-pointing double arrows or outward-pointing double arrows $(<>$; employed as free-choice targets). The stimuli subtended a visual angle of $1.15^{\circ} \times 0.4^{\circ}$. The masks were constructed from a $6 \times$ 5 matrix $\left(1.4^{\circ} \times 0.9^{\circ}\right)$, randomly filled with overlapping lines of different lengths $\left(0.06^{\circ}-0.3^{\circ}\right.$; width, $\left.0.06^{\circ}\right)$ and orientations. A different random mask was constructed on each trial. The stimuli were presented black on white on a computer screen. The primes and masks were presented at the screen center; the targets appeared randomly and with equal probability $2.5^{\circ}$ above or below the center, just outside the area occupied by the mask.

Procedure. The participants sat in a dimly lit chamber, facing a computer screen at a viewing distance of $100 \mathrm{~cm}$. Left- or rightpointing arrow primes (presented randomly and with equal probability) were followed immediately by a $100-\mathrm{msec}$ mask. In the pure free-choice and mixed tasks, prime duration was $16 \mathrm{msec}$, target duration was $100 \mathrm{msec}$, and intertrial interval was $1,300 \mathrm{msec}$. When the prime-target ISI was $0 \mathrm{msec}$, the targets appeared simul- 
taneously with the masks. When the ISI was $150 \mathrm{msec}$, the targets appeared $50 \mathrm{msec}$ after mask offset. In forced-choice blocks, prime duration was varied, and no targets were presented.

The experiment started with two pure free-choice blocks (FreeA, 40 trials per block), one employing a 0 -msec ISI and one a $150-\mathrm{msec}$ ISI. Order of blocks was balanced across participants. In $90 \%$ of all trials, the masked primes were followed by a free-choice target. In the remaining $10 \%$ catch trials, no target was presented. The participants were instructed to maintain central eye fixation, and to respond "randomly" with a left or right keypress to free-choice targets. They were strongly discouraged from employing systematic response strategies and from responding on catch trials. Several practice blocks (16 trials each) were delivered to familiarize the participants with these task requirements. After completion of FreeA, the participants were asked whether they had noticed any stimuli apart from the masks and targets. They were then informed about the primes and were specifically asked whether they had noticed their presence.

Next, the participants performed a forced-choice block $(F C 1,60$ trials), containing 30 trials with $16-\mathrm{msec}$ primes and 10 trials each with 32-, 48-, and 64-msec primes, in random order. Each trial started with a $250-\mathrm{msec}$ warning stimulus (a $0.1^{\circ} \times 0.1^{\circ}$ square), followed after $1,500 \mathrm{msec}$ by a prime and a mask. The participants were informed that a prime would be present on each trial and were instructed to press the key spatially corresponding to its direction and to "just guess" if no prime was perceived. Five hundred milliseconds after the identification response, a 4-point confidence rating scale ("1-2-3-4," with the words not confident and confident below the numbers " 1 " and " 4 ," respectively) was displayed in the screen center until a response had been given. The participants rated how confident they felt about the accuracy of their identification response by pressing one out of four response keys (placed under the left and right middle and index fingers) spatially corresponding to the selected number. Orientation of the scale (" $1-2-3-4$ " or " $4-3-2-1$ ") was varied randomly between trials.

The main part of the experiment consisted of eight blocks ( 60 trials each) that contained randomly intermixed instructed and freechoice trials (mixed). The participants had to respond as quickly and accurately as possible with a left or right keypress to left- or right-pointing arrow targets and to spontaneously and rapidly give a left or right response to free-choice targets. Instructions emphasized "not to think" about the free responses, and the practice block at the beginning of this part was repeated (up to three times) until RT differences between instructed trials and free trials were no larger than $50 \mathrm{msec}$. Mixed blocks contained one-third instructed compatible trials (prime and arrow target mapped to the same response), one-third instructed incompatible trials (prime and arrow target mapped to opposite responses), and one-third free trials (prime followed by free-choice target), in random order. Prime-target ISI was $0 \mathrm{msec}$ in four blocks and $150 \mathrm{msec}$ in the other four, with block sequence counterbalanced across participants. Finally, the participants completed another two blocks of pure free-choice trials (FreeB; identical to FreeA) and another forced-choice block (FC2, identical to FC1).

Data analysis. No data-trimming procedures were employed. Percentages of correct responses in forced-choice trials were transformed into $d^{\prime}$ values (Gescheider, 1997), and average confidence ratings were calculated for correct and incorrect responses, separately for each prime duration. For instructed trials, mean RTs for correct responses and the percentages of incorrect responses were calculated. For free trials, the percentages of congruent responses (responses corresponding to the prime arrows' direction) and mean RTs on congruent and noncongruent responses were computed separately for FreeA, mixed, and FreeB blocks. In all the analyses, Greenhouse-Geisser adjustments to the degrees of freedom were performed where appropriate (indicated by $\varepsilon$ ), and corrected $p$ values are reported.

\section{Results}

Three participants were excluded from analysis because of failure to comply with task instructions (false alarm rate of $75 \%$ in at least one free-only block).

Forced choice. On questioning, all the participants denied noticing prime stimuli in the preceding FreeA blocks, even after being informed about their presence. Identification performance (Table 1) was analyzed using an analysis of variance (ANOVA) with part (FC1 and FC2) and prime duration (16, 32, 48, and $64 \mathrm{msec})$ as variables. Performance improved with increasing prime duration $\left[F(3,60)=43.67, M S_{\mathrm{e}}=0.64, p<.001 ; \varepsilon=\right.$ .694], but did not differ between FC1 and FC2 (main effect of part, interaction of part and prime duration: both $F_{\mathrm{S}}<0.9$, both $p \mathrm{~s}>.37$ ). Subsequent $t$ tests, testing $d^{\prime}$ values against 0 , were significant for $64-$ and $48-\mathrm{msec}$ primes in both parts (all $p \mathrm{~s}<.002$ ), marginally significant for 32-msec primes in FC2 $[t(20)=1.9, p<.08]$, and not significant for 32-msec primes in FC1 and for 16-msec primes (all $p \mathrm{~s}>.17$ ).

An ANOVA on confidence ratings (Table 1) for correct responses showed that confidence increased with increasing prime duration $\left[F(3,30)=66.45, M S_{\mathrm{e}}=0.41\right.$, $p<.001 ; \varepsilon=.652]$ and did not differ between parts $(F<$ $0.25, p>.65)$. A full analysis of confidence ratings including both correct and incorrect responses was precluded by the fact that only half of all the participants produced correct and incorrect responses to primes of all the durations in both parts. It might be interesting to note, however, that statistically significant differences between ratings on correct and incorrect responses $(t \mathrm{~s}>$ $2.8, p \mathrm{~s}<.014$ ) could be obtained only for 64 -msec primes.

FreeA and FreeB. The percentage of congruent responses (Table 2A) was analyzed in an ANOVA with ISI $(0$ or $150 \mathrm{msec})$ and part (FreeA or FreeB) as variables. No significant effects were found (all $F \mathrm{~s}<1.7$, all $p \mathrm{~s}>$ .26), demonstrating that the frequency of congruent responses did not differ from chance in any block (all $p$ s $>$ .24). RTs in FreeA and FreeB were equally unaffected by congruency (Table 3 ), as is shown by the absence of any effects in an ANOVA with part, ISI, and congruency (congruent or noncongruent) as variables (all $F \mathrm{~s}<1.4$, all $p \mathrm{~s}>.25$ ). Paired $t$ tests confirmed that congruent and noncongruent RTs did not differ significantly from each other in any block (all $t \mathrm{~s}<1$, all $p \mathrm{~s}>.36$ ).

\section{Table 1}

Prime Identification Performance and Confidence Ratings on Correct Responses as a Function of Prime Duration in FC1 and FC2

\begin{tabular}{|c|c|c|c|c|c|c|c|c|}
\hline \multirow{3}{*}{$\begin{array}{c}\text { Prime } \\
\text { Duration } \\
(\mathrm{msec})\end{array}$} & \multicolumn{4}{|c|}{$d^{\prime}$} & \multicolumn{4}{|c|}{ Rating } \\
\hline & \multicolumn{2}{|c|}{$\mathrm{FC} 1$} & \multicolumn{2}{|c|}{$\mathrm{FC} 2$} & \multicolumn{2}{|c|}{$\mathrm{FCl}$} & \multicolumn{2}{|c|}{$\mathrm{FC} 2$} \\
\hline & $M$ & $S E$ & $M$ & $S E$ & $M$ & $S E$ & $M$ & $S E$ \\
\hline 64 & $1.73^{* *}$ & 0.23 & $1.99 * *$ & 0.26 & 3.50 & 0.10 & 3.53 & 0.11 \\
\hline 48 & $1.06^{* *}$ & 0.26 & $1.08 * *$ & 0.24 & 3.17 & 0.12 & 3.08 & 0.14 \\
\hline 32 & 0.18 & 0.22 & $0.35^{+}$ & 0.18 & 2.48 & 0.16 & 2.30 & 0.16 \\
\hline 16 & 0.11 & 0.08 & 0.07 & 0.10 & 1.62 & 0.15 & 1.76 & 0.16 \\
\hline
\end{tabular}

$* * d^{\prime}$ values significantly different from 0 at the $1 \%$ level. $\quad+d^{\prime}$ values approaching significance (below 10\% level). 
Table 2

Performance in Mixed and Free Blocks (Mean Percentage Prime-Corresponding Responses on Free-Choice Trials, and Mean Percent Error Rates and Error Rate Effects on Free Instructed Trials)

\begin{tabular}{|c|c|c|c|c|}
\hline \multirow[b]{3}{*}{ Trials } & \multicolumn{4}{|c|}{ Interstimulus Interval } \\
\hline & \multicolumn{2}{|c|}{$0 \mathrm{msec}$} & \multicolumn{2}{|c|}{$150 \mathrm{msec}$} \\
\hline & $M$ & $S E$ & $M$ & $S E$ \\
\hline \multicolumn{5}{|c|}{ Free-Choice Trials } \\
\hline FreeA & 47.54 & 2.43 & 50.24 & 1.92 \\
\hline FreeB & 50.39 & 1.57 & 52.02 & 1.67 \\
\hline Mixed free & $53.22 *$ & 1.44 & $46.58 *$ & 1.25 \\
\hline \multicolumn{5}{|c|}{ Free Instructed Trials } \\
\hline Compatible & 6.3 & 1.3 & 9.6 & 1.1 \\
\hline Incompatible & 14.5 & 1.1 & 8.0 & 0.9 \\
\hline Difference & $8.3 * *$ & 1.6 & $-1.6^{+}$ & 0.9 \\
\hline
\end{tabular}

${ }^{+}$Indicates effects approaching significance (below 10\% level). $\quad *$ Indicates effects significant at the $5 \%$ level. $* *$ Indicates effects significant at the $1 \%$ level.

The false alarm rate on catch trials was higher in ISI-0 blocks $(16.1 \%, S E=2.3)$ than in ISI-150 blocks [6.0\%, $\left.S E=1.6 ; F(1,20)=10.91, M S_{\mathrm{e}}=197.17, p<.005\right]$, presumably reflecting unspecific response activation triggered by the masked prime, resulting in inappropriate responses when a target was expected to occur simultaneously with the mask (ISI-0). When a target was expected after mask offset (ISI-150), unspecific response activation would already have decayed. False alarm rate did not differ between parts (main effect and interaction: both $F_{\mathrm{s}}<2.3$, both $p \mathrm{~s}>$.14).

Mixed blocks. RTs were analyzed using an ANOVA with ISI, trial (instructed or free), and compatibility/ congruency (compatible or incompatible for instructed trials, congruent or noncongruent for free trials) as variables. RTs were shorter on instructed than on free-choice trials $\left[F(1,20)=52.41, M S_{\mathrm{e}}=915.60, p<.001\right]$ and were shorter in ISI-150 blocks than in ISI-0 blocks $\left[F(1,20)=11.44, M S_{\mathrm{e}}=1,715.97, p=.003\right){ }^{2}$ The latter difference was more pronounced for instructed than for free-choice trials $\left[F(1,20)=5.95, M S_{\mathrm{e}}=223.98\right.$, $p=.024]$.
Responses were faster on compatible/congruent trials than on incompatible/noncongruent trials $[F(1,20)=$ $\left.24.31, M S_{\mathrm{e}}=133.21, p<.001\right]$. Importantly, this effect was qualified by a significant ISI $\times$ compatibility interaction $\left[F(1,20)=45.11, M S_{\mathrm{e}}=331.65, p<.001\right]$. Subsequent paired $t$ tests confirmed PCEs for both instructed and free responses in ISI-0 blocks, with shorter RTs on compatible (congruent) than on incompatible (noncongruent) trials and NCEs for both instructed and free responses in ISI-150 blocks, with shorter RTs on incompatible than on compatible trials (all $p$ s $<.018$; see Table 3 ). No trial $\times$ ISI $\times$ compatibility interaction was present $(F<$ $0.5)$, thereby further confirming that RT priming effects for instructed and free responses were very similar. Response errors on instructed trials showed a similar pattern (Table 2B), with more errors on incompatible trials in ISI-0 blocks $(p<.001)$ and a nonsignificant tendency toward more errors on compatible trials in ISI- 150 blocks $(p<.09)$.

Importantly, masked primes affected not only the speed of free responses in mixed blocks, but also the frequency with which congruent responses were chosen (Table 2A). In ISI-0 blocks, the frequency of congruent responses was above chance level $[t(20)=2.24, S E=1.44, p=$ $.037]$. In ISI-150 blocks, in contrast, congruent responses were chosen less frequently than would be expected if these choices had been entirely random $[t(20)=2.74$, $S E=1.25, p=.013]$.

\section{Discussion}

Previous masked priming studies have shown that subliminally presented information can influence responses to subsequent targets in line with currently active S-R mappings (e.g., Dehaene et al., 1998; Eimer \& Schlaghecken, 1998). Masked primes that match the currently active task set trigger an activation-followed-by-inhibition process, resulting in PCEs with a short prime-target ISI and NCEs with a longer ISI. The results observed here for instructed targets replicated these findings. Also in line with earlier results, prime identification performance confirmed that masked primes presented for $16 \mathrm{msec}$ are below the objective identification threshold and that

Table 3

Mean RTs (in Milliseconds) on Congruent and Noncongruent Free Trials in Free Blocks and Mixed Blocks, and on Compatible and Incompatible Instructed Trials in Mixed Blocks, Plus RT Differences Between Priming Conditions

\begin{tabular}{|c|c|c|c|c|c|c|c|c|c|c|c|c|}
\hline & \multicolumn{6}{|c|}{0 -msec ISI } & \multicolumn{6}{|c|}{ 150-msec ISI } \\
\hline & \multicolumn{2}{|c|}{$\begin{array}{l}\text { Congruent/ } \\
\text { Compatible }\end{array}$} & \multicolumn{2}{|c|}{$\begin{array}{l}\text { Noncongruent/ } \\
\text { Incompatible }\end{array}$} & \multicolumn{2}{|c|}{ Difference } & \multicolumn{2}{|c|}{$\begin{array}{l}\text { Congruent/ } \\
\text { Compatible }\end{array}$} & \multicolumn{2}{|c|}{$\begin{array}{l}\text { Noncongruent/ } \\
\text { Incompatible }\end{array}$} & \multicolumn{2}{|c|}{ Difference } \\
\hline & $M$ & $S E$ & $M$ & $S E$ & $M$ & $S E$ & $M$ & $S E$ & $M$ & $S E$ & $M$ & $S E$ \\
\hline FreeA & 329 & 10 & 327 & 12 & -2 & 6 & 355 & 11 & 352 & 12 & -3 & 4 \\
\hline FreeB & 352 & 14 & 355 & 15 & 3 & 6 & 350 & 13 & 345 & 12 & -5 & 5 \\
\hline Mixed free & 423 & 14 & 448 & 12 & $25 * *$ & 4 & 413 & 9 & 403 & 9 & $-10^{*}$ & 4 \\
\hline Mixed instructed & 381 & 10 & 411 & 9 & $30 * *$ & 4 & 385 & 8 & 375 & 8 & $-10^{*}$ & 3 \\
\hline
\end{tabular}

Note-All values are rounded to the nearest millisecond. *Priming effects significantly different from 0 at the $5 \%$ level. **Priming effects significantly different from 0 at the $1 \%$ level. 
identification performance for these stimuli does not increase with repeated exposure.

Importantly, the present experiment demonstrates that subliminal prime stimuli are able to systematically bias a "free" choice between response alternatives. Results for free-choice targets reveal that subliminal priming effects are not restricted to responses determined by target identity but also affect apparently "free" choices between response alternatives. In line with the activationfollowed-by-inhibition account, the direction of priming effects on free-choice responses paralleled those obtained for instructed targets: Congruent responses were chosen preferentially and were executed more quickly than noncongruent responses when the prime-target ISI was $0 \mathrm{msec}$ but were chosen with less than chance frequency and were executed more slowly than noncongruent responses when the ISI was $150 \mathrm{msec}$. Thus, the prime-induced state of motor activation - an initial activation and subsequent inhibition of the response assigned to the prime-exerted a strong bias on subjectively free response decision and execution. Asked about their responses on free-choice trials, participants consistently reported being unaware of any such biasing effects and characterized their response selection unanimously as internally generated and under voluntary control.

Importantly, these effects were observed only when the primes matched the currently active task set in the mixed blocks. When they did not (free blocks), no priming effects on response choice and response latencies were found, even when an appropriate task set had been active only minutes earlier (FreeB). Instead of attributing this absence of priming effects in the free blocks to the lack of a specific task set, one could argue that the "pure" freechoice task was less demanding and, thus, required less attentional resources than did the mixed task. Participants may simply be less susceptible to subliminal priming in the absence of focused attention. This alternative interpretation is inconsistent with results from previous studies. When masked arrow primes were followed by the letters $\mathrm{L}$ and $\mathrm{R}$ instead of arrow targets (so that arrows were not included in the current task set), no priming effects were elicited (Eimer \& Schlaghecken, 1998), although attentional demands were just as high as those in blocks including arrow targets. Under conditions in which $90 \%$ of all the trials required a response with one hand and only $10 \%$ a response with the opposite hand (thereby imposing attentional demands similar to the present free blocks, which included 10\% catch trials), subliminal priming effects were elicited (Schlaghecken \& Eimer, 2001), since primes were included in the task set. Thus, it seems reasonable to interpret the failure of priming effects in the free blocks as additional evidence for the claim that the impact of subliminal information on behavior depends on, and is mediated by, currently active intentions. Only primes that are part of the currently active task set - the set of S-R mappings imposed by task instructions and applied by the participant - will affect the motor system. When this task set is no longer relevant, it appears to become rapidly deactivated.

It should be noted that similar results have been found in two pilot experiments employing 0 - and $150-\mathrm{msec}$ prime-target ISIs, respectively. With short ISIs, congruent responses were chosen with above-chance frequency and were executed more quickly than noncongruent responses. With long ISIs, the reverse pattern was found. In both experiments, responses in pure free-choice blocks did not show any priming effects. ${ }^{3}$

Interestingly, subliminal primes, although obviously task relevant, failed to systematically affect response decisions in forced-choice blocks. This suggests that a response bias triggered by subliminal primes is not accessible to controlled response selection processes, even when task instructions require a discriminative response based on this information.

Although it has been demonstrated repeatedly that subliminal stimuli can bias "free" semantic and affective decisions (see Kihlstrom, 1999, for an overview), this is, to our knowledge, the first time that stimuli presented below an objective identification threshold have been shown to bias subjectively free motor responses. These effects are particularly interesting since they appear to bridge the gap between supposedly low-level, perception-based subliminal influences (e.g., Mandler, Nakamura, \& Van Zandt, 1987) and influences on high-level semantic processes (e.g., Marcel, 1983). Although primes affect (lowlevel) automatic stages of motor processing, these influences are mediated by (high-level) current intentions and task set.

Concerns surrounding the phenomenon of subliminal perception have been fueled by the idea that subliminal information might manipulate people in ways that are at odds with their intentions and by fears that subjectively voluntary and free decisions might turn out to be under the control of unperceived stimuli. The results reported here indicate that subliminal stimuli will affect behavior strictly in line with current intentions and only when these intentions are activated, thus leaving little room for the possibility of malicious subliminal intervention. However, the present study also suggests that subliminal influences are indeed able to bias response decisions even when such choices appear to be internally generated and free. This is, however, not necessarily bad news: Because these biases will still be in line with current intentions, their contribution to the choice of action should generally be appropriate and adaptive.

\section{REFERENCES}

Aron, A. R., Schlaghecken, F., Fletcher, P. C., Bullmore, E. T., Eimer, M., Barker, R., SAhaKian, B. J., \& Robbins, T. W. (2003). Inhibition of subliminally primed responses is mediated by the caudate and thalamus: Evidence from functional MRI and Huntington's disease. Brain, 126, 713-723.

Cheesman, J., \& Merikle, P. M. (1986). Distinguishing conscious from unconscious perceptual processes. Canadian Journal of Psychology, 40, 343-367. 
Dehaene, S., Naccache, L., Le Clec'H, G., Koechlin, E., Mueller, M., Dehaene-Lambertz, G., van De Moortele, P.-F., \& Le Bihan, D. (1998). Imaging unconscious semantic priming. $\mathrm{Na-}$ ture, 395, 597-600.

EIMER, M. (1999). Facilitatory and inhibitory effects of masked prime stimuli on motor activation and behavioral performance. Acta Psychologica, 101, 293-313.

EIMER, M., \& SCHLAGHECKEN, F. (1998). Effects of masked stimuli on motor activation: Behavioral and electrophysiological evidence. Journal of Experimental Psychology: Human Perception \& Performance, 24, 1737-1747.

Eimer, M., \& Schlaghecken, F. (2002). Links between conscious awareness and response inhibition: Evidence from masked priming. Psychonomic Bulletin \& Review, 9, 514-520.

Eimer, M., Schubö, A., \& SCHLAGHeCKen, F. (2002). Locus of inhibition in the masked priming of response alternatives. Journal of Motor Behaviour, 34, 3-10

Gescheider, G. A. (1997). Psychophysics: The fundamentals (3rd ed.). Mahwah, NJ: Erlbaum.

Kinlstrom, J. F. (1999). The psychological unconscious. In L. A. Pervin \& O. P. John (Eds.), Handbook of personality: Theory and research (2nd ed., pp. 424-442). New York: Guilford.

KLAPP, S. T., \& HinKLeY, L. B. (2002). The negative compatibility effect: Unconscious inhibition influences reaction time and response selection. Journal of Experimental Psychology: General, 131, 255269.

Leuthold, H., \& Kopp, B. (1998). Mechanisms of priming by masked stimuli: Inferences from event-related brain potentials. Psychological Science, 9, 263-269.

MANDLER, G., NAKAMURA, Y., \& VAN ZANDT, B. J. S. (1987). Nonspecific effects of exposure on stimuli that cannot be recognized. Journal of Experimental Psychology: Learning, Memory, \& Cognition, 13, 646-648.

MARCEL, A. J. (1983). Conscious and unconscious perception: Experiments on visual masking and word recognition. Cognitive Psychology, 15, 197-237.

NEUMANN, O., \& KLOTZ, W. (1994). Motor responses to nonreportable, masked stimuli: Where is the limit of direct parameter specification? In C. Umiltà \& M. Moscovitch (Eds.), Attention and performance $X V$ : Conscious and nonconscious information processing (pp. 123150). Cambridge, MA: MIT Press, Bradford Books.

Schlaghecken, F., \& Eimer, M. (1997). The influence of subliminally presented primes on response preparation. Sprache \& Kognition, 16, 166-175.

Schlaghecken, F., \& Eimer, M. (2000). A central-peripheral asymmetry in masked priming. Perception \& Psychophysics, 62, 1367-1382.

SChlagheCKen, F., \& EIMER, M. (2001). Partial response activation to masked primes is not dependent on response readiness. Perceptual \& Motor Skills, 92, 208-222.

SchlagheCKEN, F., \& EIMER M. (2002). Motor activation with and without inhibition: Evidence for a threshold mechanism in motor control. Perception \& Psychophysics, 64, 148-162.

\section{NOTES}

1. The term subliminal has been used to refer to stimuli presented below a subjective threshold of conscious awareness, below objective identification threshold, or below objective detection threshold (see Cheesman \& Merikle, 1986). For the present discussion, the first, most lenient interpretation would be sufficient. However, previous forcedchoice performance tests have shown 16-msec masked primes to be below objective threshold. In forced-choice present/absent tasks (Eimer \& Schlaghecken, 1998, Experiments 2 and 3), forced-choice identification tasks with arrow targets following masked primes (Schlaghecken \& Eimer, 1997), forced-choice identification tasks without subsequent targets (Eimer \& Schlaghecken, 1998, Experiment 1), and staircase procedures (Eimer \& Schlaghecken, 2002), participants consistently failed to perform with better-than-chance accuracy.

2. This RT advantage for longer ISIs has been observed before (e.g., Schlaghecken \& Eimer, 2000) and probably reflects an increase in response readiness with a longer foreperiod between masked primes, acting as warning stimuli, and targets.

3 . With a 0 -msec prime-target ISI, congruent responses were chosen on $56.3 \%$ of all free-choice trials $[t(8)=3.60, S E=1.7, p=.007]$, and were $16 \mathrm{msec}$ faster than noncongruent responses $[t(8)=2.78, S E=$ $5.6, p=.024]$. With 150 -msec prime-target ISI, congruent responses were chosen on $42.3 \%$ of all the trials $[t(10)=3.55, S E=2.2, p=$ $.005]$ and were $14 \mathrm{msec}$ slower than noncongruent responses $[t(10)=$ $3.1, S E=4.87, p<.013]$. No such effects were obtained in pure freechoice blocks, either before or after the mixed blocks (all $t \mathrm{~s}<2.0$, all $p \mathrm{~s}>.07)$.

(Manuscript received September 28, 2001; revision accepted for publication May 13, 2003.) 